\title{
Visualization Performance and Rapid Prototyping in First Year Design
}

Visualization literature suggests that solid-modeling software tools improve visualization skills as a result of interaction with the model. As an extension of these findings, the authors proposed that product dissection and real-time simulation of the model should further improve visualization. Our prior study examined visualization performance using solid-modeling in three teaching modalities of a Freshmen Design and Graphics course: 1) Traditional, 2) Project Based Dissection, and 3) Simulation Based Design. The first and second modalities focused assessment on the part/assembly form, whereas the third modality transitioned the outcome expectations to understanding and function of mechanism design. Our comparison between teaching modalities revealed an unexpected bifurcation of results where the top performing group from several years of data demonstrated slight performance improvement with consistent variance, while the lower performing group decreased in performance with substantial increase in variance. It was proposed that the abstract nature of mentally reconstructing a solid-model or realtime simulation was a potential factor in the lower performing group. In September 2013 the author implemented rapid prototyping capability as pilot study in improving the traditional Design \& Graphics course. This paper will discuss the implementation of rapid prototyping and present the preliminary results on visualization performance. This paper will be of interest to educators interested in first year Design \& Graphics.

Keywords: Visualization, Rapid Prototyping, Project Based Learning, Design and Graphics, First Year Engineering 